\title{
Photometry of Irregular Satellites of Uranus and Neptune
}

\author{
Tommy Grav ${ }^{1}$ \\ Harvard-Smithsonian Center for Astrophysics, MS51, 60 Garden Street, Cambridge MA \\ 02138 \\ tgrav@cfa.harvard.edu \\ Matthew J. Holman ${ }^{2}$ \\ Harvard-Smithsonian Center for Astrophysics, MS51, 60 Garden Street, Cambridge, MA 02138 \\ mholman@cfa.harvard.edu \\ Wesley C. Fraser ${ }^{2}$ \\ McMaster University, Hamilton, ON L8S 4M1, Canada \\ fraser@physics.mcmaster.ca
}

\begin{abstract}
We present BVR photometric colors of six Uranian and two Neptunian irregular satellites, collected using the Magellan Observatory (Las Campanas, Chile) and the Keck Observatory, (Manua Kea, Hawaii). The colors range from neutral to light red, and like the Jovian and the Saturnian irregulars (Grav et al. 2003a) there is an apparent lack of the extremely red objects found among the Centaurs and Kuiper belt objects.

The Uranian irregulars can be divided into three possible dynamical families, but the colors collected show that two of these dynamical families, the Caliban and Sycorax-clusters, have heterogeneous colors. Of the third possible family, the 168-degree cluster containing two objects with similar average inclinations but quite different average semi-major axis, only one object (U XXI Trinculo) was observed. The heterogeneous colors and the large dispersion of the average orbital elements leads us to doubt that they are collisional families. We favor single
\end{abstract}

\footnotetext{
${ }^{1}$ Visiting Astronomer, Keck Observatory

${ }^{2}$ Visiting Astronomer, Magellan Observatory
} 
captures as a more likely scenario. The two neptunians observed (N II Nereid and S/2002 N1) both have very similar neutral, sun-like colors. Together with the high collisional probability between these two objects over the age of the solar system (Nesvorný et al. 2003; Holman et al. 2004), this suggests that S/2002 N1 be a fragment of Nereid, broken loose during a collision or cratering event with an undetermined impactor.

Subject headings: planets and satellites

\section{Introduction}

Irregular satellites are small bodies that orbit their parent planets in large, eccentric orbits with high inclinations relative to the planet's equatorial plane. Irregular satellites have been discovered around all the giant planets and are thought to have been captured from heliocentric orbits during the last stages of the formation of the giant planets. While objects in heliocentric orbits may be temporarily captured in planetocentric orbits, some loss of energy is needed to make the capture permanent. Several processes have been proposed for this change of orbital energy: 1) an increase in the mass of the planet through accretion (Heppenheimer \& Porco 1977); 2) gas drag through an extended envelope or disk around the still forming planet (Pollack et al. 1979); 3) collision or close encounters with a preexisting regular moon or another temporarily captured object (Colombo \& Franklin 1971); 4) dynamical friction from a large number of small outer solar system bodies (Astakhov et al. 2003; Goldreich et al. 2002).

It was recognized by Colombo \& Franklin (1971), and later by Gladman et al. (2001), that the irregular satellites cluster in groups with similar dynamical properties. This clustering is considered to be evidence that the members of a cluster are remnants of a larger progenitor that was captured and subsequently broken up. In Grav et al. (2003a) we reported optical BVRI photometry of a large number of irregular satellites of Jupiter and Saturn, showing that almost all of the known dynamical clusters have homogeneous colors, supporting the thesis suggesting the fragmentation of larger progenitors. Determining the near-infrared colors of the brightest Jovian and Saturnian irregular satellites revealed that the normalized broadband reflectance spectra are very similar to the spectra of C- and D-type outer main belt asteroids (Grav \& Holman 2004).

We now turn to Uranus and Neptune. The physical knowledge of the irregular satellites of the two outer planets is extremely limited. Only two Uranian and one Neptunian irregular satellites have been the targets of photometrical studies and have had their colors determined. 
Maris et al. (2001) observed the two Uranian satellites, U XVI Caliban and U XVII Sycorax, in BVRI filters, using the $3.6 \mathrm{~m}$ ESO NTT, on La Silla, Chile. They found that both have moderately red colors, with Sycorax being slightly bluer than Caliban. They compared their observations to other families of objects in the solar system and found that the two satellites were clearly redder than Uranus and its regular satellites. They also compared the $V-R$ colors with the histogram found in Jewitt et al. (1996) and placed the satellites among the bluest Kuiper belt objects and the reddest near-earth objects.

Romon et al. (2001) performed a more detailed study of Sycorax, also determining the BVRI colors using the $3.5 \mathrm{~m}$ Telescopio Nazionale Galileo, La Palma. They, however, added photometric observations in the J-band and spectroscopic observations in the near-infrared using ESO's 8m Very Large Telescope in Chile. They compared their results with that of other small bodies in the Solar System, and argued that Sycorax is more similar to TNOs, Centaurs and cometary nuclei, than to the Trojans and irregular satellites of Jupiter.

N II Nereid was discovered in 1949 by G. Kuiper. Due to its brightness it has been extensively studied. Colors have been determined by Schaefer \& Schaefer (2000), and Brown et al. (1999) used near-infrared spectra to show that the satellite has features indicative of water ice. Voyager II observations during its fly-by of Neptune were used to determine the albedo, $p \sim 0.2$, of Nereid (Thomas et al. 1991). It is thus believed that Nereid is an icy body. It is either an inner satellite gravitationally scattered as N I Triton was captured or a captured ice body originating in the Kuiper belt (McKinnon 1984; Goldreich et al. 1989).

In this paper we report the BVR colors of six Uranian and two Neptunian irregular satellites, and discuss the implications of the colors determined. In section 2 we describe the observations performed and our method of data reduction. Section 3 contains the results and interpretations of the observations.

\section{The Observations}

The observations presented here were performed at the $6.5 \mathrm{~m}$ Clay telescope at the Magellan Observatory using the MagIC instrument and at the $10 \mathrm{~m}$ Keck II telescope using the DEIMOS instrument. The observations at the Clay telescope were performed on July 27th and 28th, 2003. The conditions were photometric and observations of Caliban, Sycorax, U XX Stephano, Nereid and S/2002 N1 were performed . The observations using the Keck were done on August 1, 2003. The first part of the night was subject to high cirrus clouds, that moved off to the horizon at about midnight. The remainder of the night was photometric and used to observe the uranian satellites U XVIII Prospero, U XIX Setebos and 
U XXI Trinculo, as well as the neptunian satellite S/2002 N1.

A number of standard stars (Landolt 1992) were observed each night, covering the same elevation as the science targets. Transformation equations containing zero points, airmass corrections and color corrections were determined and used to determine the V-magnitude and colors (B-V and V-R) of the science targets. The DAOPHOT package under the IRAF environment was used for the all the data reduction.

\subsection{Magellan MagIC}

The MagIC instrument is a single SITe 2048x2048 CCD camera with a rather small field of view, 2 by 2 arcminutes. For the MagIC observations a Harris BVR filter set was used. The seeing during the observations varied from $1.2-2.0$ arcseconds. We performed aperture photometry using a inner and outer aperture of 1.725 and 4.14 arcseconds, respectively. The aperture correction were small (0.05- 0.21 magnitudes) in all filters. Using the observed Landolt standars stars we determined the following transformation equations:

$$
\begin{aligned}
& B=b+26.80-0.22 a \\
& V=v+26.94-0.21 a \\
& R=r+27.15-0.15 a
\end{aligned}
$$

where $b, v$ and $r$ are the instrumental magnitudes and $a$ is the airmass. Color corrections were included in the determination of the transformation equations, but were negligible.

\section{2. $\quad$ Keck II DEIMOS}

The DEep Imaging Multi-Object Spectrograph (DEIMOS) is an optical wavelength imaging spectrograph. We used the instrument in direct imaging mode. The seeing varied from $0.8-1.2$ arc-seconds. We again used aperture photometry, using an inner and outer aperture of 0.95 and 2.37 arc-seconds, respectively. The aperture corrections were also small (0.05 - 0.29 magnitudes) in all filters.

The filters available for the DEIMOS are rather special and do not conform to any of the usual filter systems. We therefore included color corrections in the determination of the transformation equations from instrumental to photometric magnitude. The color corrections in the B and V filters were non-negligible but still small given the moderate colors of our targets. For the Keck II observations we derived the following transformation 
equations:

$$
\begin{aligned}
& B=b+27.33-0.24 a+0.23(B-V) \\
& V=v+27.90-0.24 a-0.15(B-V) \\
& R=r+28.06-0.15 a
\end{aligned}
$$

where, again, $b, v$ and $r$ are the instrumental magnitudes and $a$ is the airmass.

\section{The Results}

The data collected is presented in Table 1 and plotted in a standard B-V vs V-R diagram in Figure 1. The colors are similar to those found among the Jovian and Saturnian, and the colors seem to be separated into two groups. One is essentially neutrally colored containing Prospero, Setebos, the two Neptunians observed, and possibly Trinculo. The other is slighty red and contains the two large Uranians, Caliban and Sycorax. The Uranian irregular Stephano may, due to its large error bars, be put in either of the two groups. Interestingly, neither the Uranian or the Neptunian irregular satellites have members with the extremely red colors found among the Kuiper belt objects.

Caliban, Sycorax, and Nereid have had their colors determined previously (Schaefer \& Schaefer 2000; Maris et al. 2001; Romon et al. 2001). The colors determined in this paper is in excellent agreement with the results of these papers, except for the B-V colors of Caliban and Sycorax determined by Maris et al. (2001). Their B-V colors are higher than and inconsistent with ours even at the $3 \sigma$ level. We are unable to explain this discrepancy.

Using the geometric circumstances of the observations (see Table 2) we have used the observed V-magnitude to derive absolute magnitudes at zero phase angle and unit heliocentric and geocentric distances. The equation used is

$$
m_{V}(1,1,0)=V-5 \log (\Delta r)-\beta \alpha
$$

where $\mathrm{V}$ is the observed magnitude, $\alpha$ is the phase angle, $\beta$ is the phase angle correction factor, and $\Delta$ and $r$ are the geocentric and heliocentric distances, respectively. While the distances and the phase angle are well known quantities, the linear phase coefficient, $\beta$, is highly uncertain. Schaefer \& Tourtellotte (2001) observed Nereid at a range of phase angles and and found $\beta=0.38$ for $\alpha<1^{\circ}$. This value is similar to the phase coefficients of small, inner Uranian satellites (Karkoschka 2001). We will use this value to estimate the absolute magnitudes for both the Uranian and Neptunian irregulars. We use the derived absolute 
magnitudes to determine the sizes of the satellites, by applying the equation:

$$
D=\frac{1329 \cdot 10^{\left[-m_{V} / 5\right]}}{\sqrt{p}}
$$

where $p$ is the geometric albedo of the satellite in the visual. The geometric albedo of the irregular satellites is another highly unknown property. Of the Uranian and Neptunian only Nereid has had its albedo determined at $p=0.2$ (Thomas et al. 1991). This value is significantly larger than that of the small inner neptunians $(p=0.06)$, the small inner Uranian satellites $(p=0.07)$, J VI Himalia $(p=0.05)$ and S IX Phoebe $(p=0.08)$. We have based on this chosen to use $p=0.07$ for the Uranian irregulars and $p=0.2$ for the Neptunian irregulars. The resulting sizes are given in Table 2.

\subsection{The Uranian Irregular Satellites}

Unfortunately, the colors of the Uranian irregular satellites offer little information about the satellites' origin. To check for dynamical families among the Uranian irregulars, we performed long-term ( $t \sim 10^{8}$ years) integrations of the nominal orbits of the known irregular satellites, and our resulting average elements are similar to those found by Nesvorný et al. (2003) (the average elements found in our integrations are plotted in Figure 2 and 3, which includes three Uranian irregulars not found in Nesvorný et al. (2003)).

From the average orbital elements we divide the known Uranian irregulars into two possible dynamical families. The Caliban-family include Caliban, Stephano and S/2001 U3, while the Sycorax-family consists of Sycorax, Prospero and Setebos. This leaves Trinculo, S/2001 U1 and S/2003 U3 (the only prograde uranian irregular satellite known to date) as single objects. It is interesting to note that Trinculo and S/2001 U1 have very similar inclinations, similar to the two prograde Saturnian irregular satellites, and could thus be a possible third dynamical family or perhaps the results of a different capturing process than the other Uranian irregulars. Their separation in average semi-major axis is significantly larger, $\Delta a=0.08 \mathrm{AU}$, than that of the Saturnian Inuit and Gallic clusters $\left(\Delta a_{I}=0.01 \mathrm{AU}\right.$ and $\Delta a_{G}=0.04 \mathrm{AU}$, respectively).

It is thus clear that if the known Uranian irregular satellites are indeed clustered into dynamical families, these two families have heterogeneous colors. It should be noted that this is not necessarily hard evidence against family structure. The Hilda asteroids, for example, have an apparent spectral slope-size, with the large members being P-types and the smaller being D-type asteroids (Dahlgren \& Lagerkvist 1995; Dahlgren et al. 1997). It is possible that the Uranian irregular satellites have a similar size-color correlation, but the low number of known objects available make this theory a pure speculation. 


\subsection{Nereid and S/2002 N1}

The observed colors of Nereid and S/2002 N1 are basically the same, suggesting similar surface compositions. Studies of the collisional probabilities between the irregular satellites of Neptune show that Nereid and S/2002 N1 has a high probability of colliding ( 0.41) over 4.5 Gy (Holman et al. 2004). The similar colors of these two objects thus point to the possibility that S/2002 N1 is a fragment of Nereid. The nearly spherical shape of Nereid (Grav et al. 2003b) offers no photometric evidence that Nereid was ever catastrophically disrupted, however Voyager II images suggest a cratered body (Thomas et al. 1991). We suggest that S/2002 N1 is therefore ejecta from cratering event on Nereid. This hypothesis would be further supported if smaller irregular satellites with similar colors and high collisional probabilities with Nereid were found. The neutral colors of Nereid and S/2002 N1 does not give any new hint to the possible origins of the two satellites. The colors, albedo and spectra of Nereid are similar to both regular satellites such as Oberon and Umbriel (Buratti \& Mosher 1991; Brown et al. 1999), as well as several Kuiper belt objects (Tegler et al. 2003).

The large difference in magnitude between the two observations of S/2002 N1 indicates that the object has a significant rotational light curve, most likely due to an out-of-round shape. It is also possible that the object has a significant opposition surge in its phase angle light curve. Nereid has a significant opposition surge (Schaefer \& Tourtellotte 2001), with an increase of 0.38 magnitudes per degree. If the magnitude difference of S/2002 N1 found in this paper is solely due to an opposition effect it implies an increase of 3.9 magnitudes per degree, a full order of magnitude larger than that of Nereid. This seems extremely unlikely, leading us to believe that the magnitude difference is fully or partially due to a rotational light curve.

\section{Conclusions}

We have reported $B V R$ colors of six Uranian and two Neptunian irregular satellites. The colors are similar to that of the Jovian and Saturnian irregular satellites (Grav et al. 2003a). It seems however unlikely that the irregular satellites of Uranus and Neptune have origins in the outer main asteroidal belt. The lack of extremely red objects among the observed

Uranian and Neptunian irregulars makes their color distribution different from that of the Centaurs and Kuiper belt, thus hinting at an origin among the outer planets. Note that the lack of extremely red objects could just be due to the small sample of objects studied. Further study of the irregular satellite population should settle this issue.

The heterogeneity of the possible dynamical families in the uranian irregular system 
leads us to question the veracity of the apparent dynamical families. With both Uranus and Neptune possibly having undergone special events (the Great Collision (Brunini et al. 2002) and the capture of Triton (Farinella et al. 1980)), it seems uncertain whether one would expect any dynamical families of irregular satellites to exist around the two planets. If one chooses to invoke the hypothesis that the Caliban- and Sycorax-clusters are indeed real families, one has to favor the theory that the similar inclinations of Trinculo and S/2001 U1 are due to a different capturing process than the rest of the uranian irregulars, due to the very large separation of semi-major axis of the two objects. Further study of this is necessary.

\section{Acknowledgments}

Some of the data presented herein were obtained at the W.M. Keck Observatory, which is operated as a scientific partnership among the California Institute of Technology, the University of California and the National Aeronautics and Space Administration. The Observatory was made possible by the generous financial support of the W.M. Keck Foundation.

Tommy Grav is a Smithsonian Astrophysical Observatory Pre-doctoral Fellow at the Harvard-Smithsonian Center for Astrophysics, Cambridge, USA. He is a graduate student at the Institute for Theoretical Astrophysics at the University in Oslo, Norway.

This work was supported by NASA grants JPL1257944, NAG5-9678 and NAG5-10438.

\section{Figure and Table Captions}

\section{Figure 1.}

B-V vs. V-R color. The error bars show the 1- $\sigma$ errors. The dashed line is the the same line

used to arbitrarily separate the Jovian and Saturnian irregular satellites into the gray and light red in Grav et al. (2003a).

Figure 2.

The average semi-major axis $(a)$ and eccentricities $(e)$ of the irregular satellites of Uranus from $10^{8}$ year integrations. The lines indicate possible irregular satellite families

\section{Figure 3.}

The average semi-major axis $(a)$ and inclinations $(i)$ of the irregular satellites of Uranus from $10^{8}$ year integrations. The lines indicate possible irregular satellite families.

\section{Table 1.}

The results of our $B V R$ color survey of the Uranian and Neptunian. The table gives the $1 \sigma$ 
errors. The first line for S/2002 N1 gives the weighted mean values of the two observations performed.

\section{Table 2.}

The geometry of the observations. The table gives the heliocentric distance, $r$, geocentric distance, $\Delta$ and phase angle, $\alpha$. The table also shows the derived absolute magnitude at opposition, unit heliocentric and geocentric distance, as well as the derived sizes.

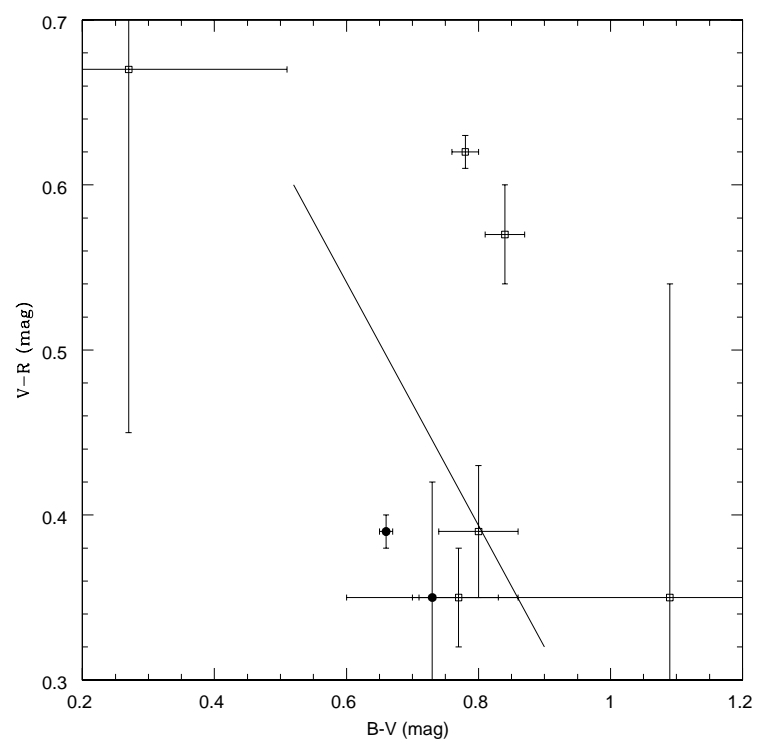

Fig. 1.- Grav, Holman \& Fraser (2004) 


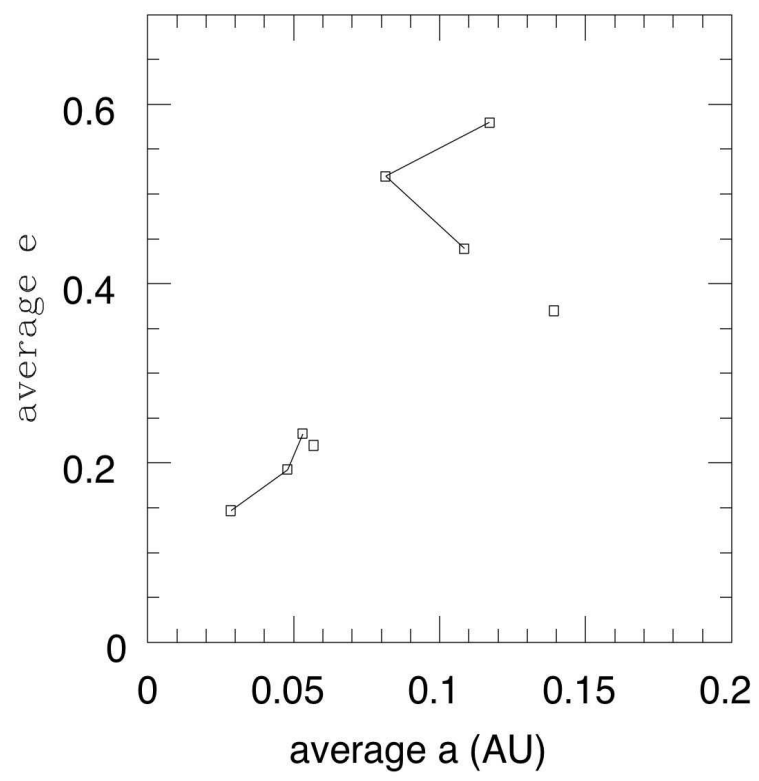

Fig. 2.- Grav, Holman \& Fraser (2004)

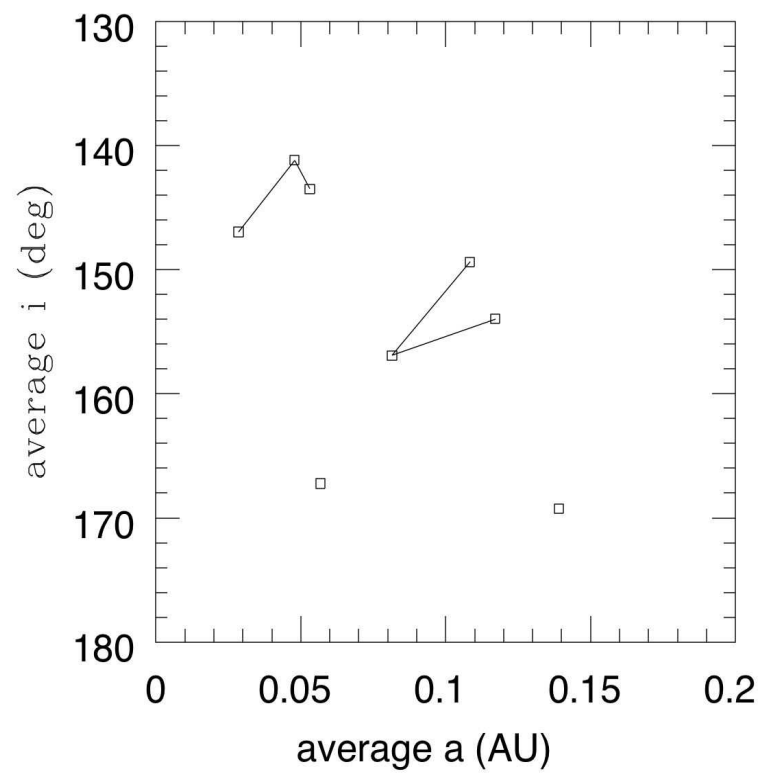

Fig. 3.- Grav, Holman \& Fraser (2004) 


\begin{tabular}{lccc} 
Object & $\mathrm{V}$ & $\mathrm{B}-\mathrm{V}$ & $\mathrm{V}-\mathrm{R}$ \\
\hline U XVI Caliban & $22.58 \pm 0.02$ & $0.84 \pm 0.03$ & $0.57 \pm 0.03$ \\
U XVII Sycorax & $20.94 \pm 0.01$ & $0.78 \pm 0.02$ & $0.62 \pm 0.01$ \\
U XVIII Prospero & $23.91 \pm 0.03$ & $0.80 \pm 0.06$ & $0.39 \pm 0.04$ \\
U XIX Setebos & $23.88 \pm 0.03$ & $0.77 \pm 0.06$ & $0.35 \pm 0.03$ \\
U XX Stephano & $25.12 \pm 0.17$ & $0.27 \pm 0.24$ & $0.67 \pm 0.22$ \\
U XXI Trinculo & $25.25 \pm 0.18$ & $1.09 \pm 0.40$ & $0.35 \pm 0.19$ \\
& & & \\
N II Nereid & $19.25 \pm 0.01$ & $0.66 \pm 0.01$ & $0.39 \pm 0.01$ \\
S/2002 N1 & & $0.73 \pm 0.13$ & $0.35 \pm 0.07$ \\
& $23.82 \pm 0.06$ & $0.87 \pm 0.10$ & $0.29 \pm 0.08$ \\
& $24.48 \pm 0.09$ & $0.51 \pm 0.16$ & $0.47 \pm 0.12$
\end{tabular}

Table 1: Grav, Holman \& Fraser (2004)

\begin{tabular}{llccccc} 
Object & Telescope & $\mathrm{r}$ & $\Delta$ & $\alpha$ & $m_{V}$ & $\mathrm{D}$ \\
& & $(\mathrm{AU})$ & $(\mathrm{AU})$ & $(\mathrm{o})$ & $(1,1,0)$ & $(\mathrm{km})$ \\
\hline U XVI Caliban & Magellan & 20.02 & 19.11 & 1.33 & $9.16 \pm 0.04$ & $\sim 74$ \\
U XVII Sycorax & Magellan & 20.08 & 19.18 & 1.34 & $7.50 \pm 0.02$ & $\sim 159$ \\
U XVIII Prospero & Keck II & 20.15 & 19.20 & 1.09 & $10.56 \pm 0.05$ & $\sim 39$ \\
U XIX Setebos & Keck II & 19.96 & 19.02 & 1.10 & $10.57 \pm 0.05$ & $\sim 39$ \\
U XX Stephano & Magellan & 20.07 & 19.16 & 1.32 & $11.69 \pm 0.17$ & $\sim 23$ \\
U XXI Trinculo & Keck II & 20.07 & 19.12 & 1.08 & $11.92 \pm 0.18$ & $\sim 21$ \\
& & & & & & \\
N II Nereid & Magellan & 30.07 & 29.06 & 0.26 & $4.44 \pm 0.01$ & $\sim 384$ \\
S/2002 N1 & Magellan & 30.12 & 29.11 & 0.25 & $9.01 \pm 0.07$ & $\sim 47$ \\
& Keck II & 30.12 & 29.10 & 0.08 & $9.74 \pm 0.08$ & $\sim 34$
\end{tabular}

Table 2: Grav, Holman \& Fraser (2004) 


\section{REFERENCES}

Astakhov, S. A., Burbanks, A. D., Wiggins, S., \& Farrelly, D. 2003, Nature, 423, 264

Brown, R. H., Cruikshank, D. P., Pendleton, Y., \& Veeder, G. J. 1999, Icarus, 139, 374

Brunini, A., Parisi, M. G., \& Tancredi, G. 2002, Icarus, 159, 166

Buratti, B. J. \& Mosher, J. A. 1991, Icarus, 90, 1

Colombo, G. \& Franklin, F. A. 1971, Icarus, 15, 186

Dahlgren, M. \& Lagerkvist, C.-I. 1995, A\&A, 302, 907

Dahlgren, M., Lagerkvist, C.-I., Fitzsimmons, A., Williams, I. P., \& Gordon, M. 1997, A\&A, 323,606

Farinella, P., Milani, A., Nobili, A. M., \& Valsecchi, G. B. 1980, Icarus, 44, 810

Gladman, B., Kavelaars, J. J., Holman, M., Nicholson, P. D., Burns, J. A., Hergenrother, C. W., Petit, J., Marsden, B. G., Jacobson, R., Gray, W., \& Grav, T. 2001, Nature, 412,163

Goldreich, P., Lithwick, Y., \& Sari, R. 2002, Nature, 420, 643

Goldreich, P., Murray, N., Longaretti, P. Y., \& Banfield, D. 1989, Science, 245, 500

Grav, T. \& Holman, M. J. 2004, ApJ, 605, L141

Grav, T., Holman, M. J., Gladman, B. J., \& Aksnes, K. 2003a, Icarus, 166, 33

Grav, T., Holman, M. J., \& Kavelaars, J. J. 2003b, ApJ, 591, 71

Heppenheimer, T. A. \& Porco, C. 1977, Icarus, 30, 385

Holman, M., Kavelaars, J., Grav, T., Gladman, B. J., Fraser, W., Milisavljevic, D., Nicholson, P. D., Burns, J. A., Carruba, V., Petit, J.-M., Rousselot, P., Mousis, O., Marsden, B. G., \& Jacobson, R. A. 2004, submitted to Nature

Jewitt, D., Luu, J., \& Chen, J. 1996, AJ, 112, 1225

Karkoschka, E. 2001, Icarus, 151, 51

Landolt, A. U. 1992, AJ, 104, 372

Maris, M., Carraro, G., Cremonese, G., \& Fulle, M. 2001, AJ, 121, 2800 
McKinnon, W. B. 1984, Nature, 311, 355

Nesvorný, D., Alvarellos, J. L. A., Dones, L., \& Levison, H. F. 2003, AJ, 126, 398

Pollack, J. B., Burns, J. A., \& Tauber, M. E. 1979, Icarus, 37, 587

Romon, J., de Bergh, C., Barucci, M. A., Doressoundiram, A., Cuby, J.-G., Le Bras, A., Douté, S., \& Schmitt, B. 2001, A\&A, 376, 310

Schaefer, B. E. \& Schaefer, M. W. 2000, Icarus, 146, 541

Schaefer, B. E. \& Tourtellotte, S. W. 2001, Icarus, 151, 112

Tegler, S. C., Romanishin, W., \& Consolmagno, S. J. 2003, ApJ, 599, L49

Thomas, P., Veverka, J., \& Helfenstein, P. 1991, J. Geophys. Res., 96, 19253 